\title{
Creatine Kinase Isoenzymes in High-Risk Infants
}

\author{
RAUL A. CUESTAS JR. ${ }^{(26)}$ \\ Department of Pediatrics, Schumpert Medical Center, Shreveport, Louisiana, USA
}

\begin{abstract}
Summary
Serial measurements of serum creatine kinase isoenzymes were done from birth to 15 days of age in healthy premature infants, infants with perinatal or neonatal asphyxia and infants without asphyxia but with miscellaneous problems. Serum brain-specific fraction of creatine kinase (CK-BB) activity was higher in cord than in maternal serum $(P<0.01)$. In healthy infants, the serum activity of heart- and muscle-specific fractions of creatine kinase increased after birth, reached a plateau between 12 and $48 \mathrm{hr}$, and then declined, whereas the serum CK-BB decreased rapidly after birth and remained stable between 6 hr and 15 days. Compared to controls, infants with severe asphyxia and neurologic damage had a significant rise in serum CK-BB $(P<0.001)$. When the peak CK-BB level exceeded 35 IU/liter the mortality was high (83\%). The increase in CK-BB was not observed in infants who received pentobarbital shortly after the episode of asphyxia. Diseases of the lung, kidneys, gastrointestinal tract, and abruptio placentae were not associated with increases of serum creatine kinase isoenzymes.
\end{abstract}

\section{Speculation}

The rise in serum brain-specific fraction of creatine kinase (CKBB) reached maximal levels 5 to $15 \mathrm{hr}$ after the asphyxial insult; thus, the finding of a very high CK-BB activity in cord serum would imply intrauterine asphyxia taking place several hr before delivery, and cord CK-BB determinations could be useful for the evaluation of fetal distress. Correlation of neonatal CK-BB levels with long-term neurologic outcome would determine whether CKBB can be used for the early prediction of the extent of neurologic damage following neonatal asphyxia. CK-BB activity determinations might also be useful in assessing the efficacy of therapeutic maneuvers aimed at preventing or minimizing hypoxic-ischemic central nervous system insults.

Determination of serum creatine kinase (CK) activity in adult patients is commonly done for evaluation of disorders involving damage to the myocardium, skeletal muscle, and central nervous system. Some data $(1,2,6,13,17)$ suggest that $C K$ determinations may also be clinically useful in newborns. The purpose of this study was to evaluate serum CK isoenzymes in high-risk newborns under various clinical conditions.

\section{MATERIALS AND METHODS}

Blood was drawn by venipuncture or from umbilical artery catheters from newborns in the intensive care nursery at the following times: birth (cord blood), 6, 12, 24, 48, and $72 \mathrm{hr}$, and 5 and 15 days. In some cases, blood was also obtained from mothers in labor. We used for statistical analysis only data from infants in whom at least 4 samples were obtained. Gestational age was estimated by physical examination and obstetric history. Four groups of patients were defined initially: group 1 (controls), infants who were asymptomatic and admitted because of prematurity and infants with mild respiratory distress syndrome or transient tachypnea of the newborn who did not require mechanical ventilation. These infants had no birth asphyxia (defined as a 5-min Apgar score of 4 or less), sepsis, congenital malformations, or systemic illnesses; group 2, infants with severe respiratory problems requiring mechanical ventilation who had no history of birth asphyxia or hypoxia in the postnatal period; group 3, infants with severe asphyxia, either at delivery or later, who required mechanical ventilation and who received a short-acting barbiturate (pentobarbital). The patients in this group received pentobarbital IV ( 5 to $7 \mathrm{mg} / \mathrm{kg}$ ) within one $\mathrm{hr}$ of the hypoxic episode at the time of initiating mechanical ventilation, when it was considered clinically necessary to obtain muscular relaxation and facilitate ventilation group 4, infants who were asphyxiated or hypoxic as in group 3 but who did not receive barbiturates. Patients were classified into one of these groups according to their clinical history and before knowledge of the CK results. After the data from the above groups were analyzed, two more groups were studied. Group 5 consisted of infants with problems of the lungs, kidneys, or gastrointestinal tract or who had abruptio placentae. The rationale for this selection was the high prevelance of these problems among sick neonates and the high brain-specific fraction of creatine kinase (CKBB) content of human placenta (10), gastrointestinal tract, and kidneys (23). None of the patients in this group was asphyxiated. Group 6 consisted of patients in whom severe neonatal or perinatal asphyxia was followed by severe neurologic damage manifested as intraventricular hemorrhage. This group also included two patients in whom mechanical neuronal injury occurred due to insertion of a ventriculoperitoneal shunt.

Serum was separated and frozen in most cases within one hr after collection and analyzed within $72 \mathrm{hr}$. Total CK activity was determined with the DuPont Automatic Clinical Analyzer using a modification of the enzymatic determination described by Tietz (21). The modification includes addition of fluoride to inhibit myokinase, dithioerythritol to activate $\mathrm{CK}$, morpholinopropane sulfonic acid to increase $C K$ reactivity, and adenosinetriphosphate to reduce the blank reaction. Determination of $\mathrm{CK}$ isoenzymes was done using the Helena CPK Isoenzyme System (Helena Laboratories). The samples were electrophoresed on cellulose acetate strips for $8 \mathrm{~min}$ at $350 \mathrm{~V}$, and the strips were then incubated for $30 \mathrm{~min}$ at $37^{\circ} \mathrm{C}$ with the $\mathrm{CK}$ reagents. The relative activity of the bands was quantitated using an automated fluorescence densitometer, and the activity of each isoenzyme was calculated as the product of the fractional activity times the total CK activity. The interassay coefficients of variation with these methods are as follows: for the total CK activity, 3.6\%; for the fractional activity of each of the isoenzymes, 16.6, 8.2, and $5.5 \%$ for brain- (CK-BB), heart- (CK-MB), and muscle- (CK-MM) specific fractions of creatine kinase respectively. The lower limit of detectability for the CK activity is 2 IU/liter; for analysis of the data, activities reported as less than 2 IU/liter were arbitrarily assigned a value of $1 \mathrm{IU} /$ liter. 


\section{RESULTS}

As stated below, the CK-BB values were not normally distributed. For this reason, statistical comparisons were made using nonparametric methods. Standard deviations are reported with the means to give an idea of the variability but were not used for comparisons of samples.

\section{DATA ON CORD AND MATERNAL SERUM}

There were no significant differences in estimated gestational age (EGA) and birth weight among the six groups of patients (Table 1). The cord serum results in infants with no birth asphyxia were analyzed in relation to the following perinatal factors: birth weight, EGA, presence of abruptio placentae, and route of delivery. There was a negative correlation between birth weight and serum CK-BB at birth; infants under $1750 \mathrm{~g}$ had higher cord serum CK-BB activity $(55.3 \pm 73.0 \mathrm{IU} /$ liter $)$ than larger infants (14.1 $\pm 7.8 \mathrm{IU} /$ liter $)$. A similar trend was observed with EGA using a cutoff point at $30 \mathrm{wk}$. No correlation was found between cord serum $\mathrm{CK}$ isoenzymes and the other perinatal factors. In 13 pairs of maternal and cord serum studied, the mean CK-BB activity was significantly higher in the cord than in the maternal serum (Table 2). In five infants delivered by cesarean section before the onset of labor, the mean serum CK-BB activity (28.2 $\pm 45.4 \mathrm{IU} /$ liter) was not significantly different than the mean maternal serum CK-BB (11.0 \pm 18.1 IU/liter).

Cord serum CK-BB activity was significantly higher in group 3 than in the controls (Table 3). In group 3, the birth weight of the six infants from whom cord blood was obtained (1209 $\pm 598 \mathrm{~g})$ was significantly smaller than in the 13 corresponding controls $(1835 \pm 578 \mathrm{~g} ; P<0.05$ ), which may explain the higher cord $\mathrm{CK}$ $\mathrm{BB}$ in those patients.

Table 1. Birth weight and gestational age in the six groups of patients studied

\begin{tabular}{crlc}
\hline Group & $n^{1}$ & \multicolumn{1}{c}{$\begin{array}{c}\text { Birth wt } \\
(\mathrm{g})\end{array}$} & $\begin{array}{c}\text { Gestational age } \\
(\mathrm{wk})\end{array}$ \\
\hline I (controls) & 29 & $1879 \pm 526^{2}$ & $32.2 \pm 2.9$ \\
II & 9 & $1727 \pm 1012$ & $29.9 \pm 3.9$ \\
III & 9 & $1470 \pm 893$ & $30.2 \pm 5.1$ \\
IV & 11 & $2036 \pm 1158$ & $33.8 \pm 6.5$ \\
V & 15 & $2196 \pm 916$ & $34.6 \pm 5.4$ \\
VI & 7 & $2351 \pm 945$ & $34.6 \pm 5.4$ \\
\hline
\end{tabular}

' $n$, number of patients in sample.

${ }^{2}$ Mean \pm S.D.

Table 2. Serum CK isoenzyme activities (IU/liter) of mothers in labor and their infants at birth (cord serum) ${ }^{1}$

\begin{tabular}{lccc}
\hline & CK-BB & CK-MB & CK-MM \\
\hline Maternal & $11.0 \pm 18.1^{2}$ & $5.9 \pm 5.2$ & $173 \pm 196$ \\
Cord & $54.5 \pm 70.1^{3}$ & $13.6 \pm 13.0$ & $146 \pm 91$ \\
\hline
\end{tabular}

${ }^{1}$ Thirteen pairs of samples were studied.

${ }^{2}$ Mean \pm S.D.

${ }^{3} P<0.01$; Wilcoxon's paired sign rank test, maternal versus cord.

Table 3. Serum $C K$ isoenzyme activities (IU/liter) at birth (cord serum) in high-risk infants'

\begin{tabular}{crccc}
\hline Group & $n^{2}$ & CK-BB & CK-MB & CK-MM \\
\hline 1 & 13 & $16.5 \pm 12.5^{3}$ & $8.9 \pm 6.2$ & $137 \pm 77$ \\
2 & 7 & $80.6 \pm 87.9$ & $10.6 \pm 11.5$ & $113 \pm 53$ \\
3 & 6 & $45.8 \pm 16.8^{4}$ & $14.8 \pm 9.5$ & $129 \pm 39$ \\
4 & 9 & $45.7 \pm 45.3$ & $9.3 \pm 10.2$ & $146 \pm 90$ \\
\hline
\end{tabular}

${ }^{1}$ The groups are described in the text.

${ }^{2} n$, number of patients in sample.

${ }^{3}$ Mean \pm S.D.

${ }^{4} P<0.005$ (versus 1), Mann-Whitney test.

\section{DATA ON POSTNATAL SERUM}

Within group 1, there was no significant difference in the CK isoenzyme activities of the asymptomatic patients and those with mild respiratory symptoms. Serum CK-BB activity decreased rapidly after birth and reached a stable value by $6 \mathrm{hr}$. From $6 \mathrm{hr}$ to 15 days, the mean CK-BB activity did not change significantly, and there was no trend with age $(r=-0.04)$; for this reason, the CK-BB results during this interval were pooled (Table 4 ). The distribution of serum CK-BB activities was markedly clustered at the lower end, with $60 \%$ of the values under $4 \mathrm{IU} /$ liter and $95 \%$ under $16 \mathrm{IU} /$ liter. We designated as normal CK-BB values of 15 IU/liter or less. In contrast to CK-BB, CK-MB and CK-MM increased after birth, reached maximal levels between 12 and 24 $\mathrm{hr}$, and decreased to baseline (cord) levels at $72 \mathrm{hr}$ or later (Table 5). Serum CK-MB and CK-MM activity in groups 2 to 4 was not significantly different from the controls; however, some of the asphyxiated newborns in group 4 had extreme elevations in the serum activity of these isoenzymes.

In groups 2 and 3, CK-BB activity was similar to that of the controls (Table 4). In Group 4, the serum CK-BB activity during the postnatal period was markedly higher $(20.4 \pm 23.5 \mathrm{IU} /$ liter $)$ than in group $1(4.7 \pm 5.2 \mathrm{IU} /$ liter; $P<0.001$; Mann-Whitney test).

Group 5 consisted of three patients with necrotizing enterocolitis, one with gastroschisis repair, one with intestinal resection and closure of ileostomy, two with severe bacterial pneumonia, one with pulmonary hemorrhage, two with pulmonary edema, one with renal failure due to polycystic kidney disease, and four with abruptio placentae. One of the patients with pulmonary edema also had renal failure and bilateral renal hemorrhages. In this group, the CK isoenzymes were not significantly different from the controls. Figure 1 shows serial serum CK-BB activities in 5 patients with severe perinatal hypoxia and two patients who had insertion of ventriculoperitoneal shunts (group 6). In the patients with shunts, the initial sample was obtained $1 \mathrm{hr}$ prior to surgery. In most cases, serum CK-BB activity reached maximal values between 5 and $15 \mathrm{hr}$ after the central nervous system insult. The maximal CK-BB level varied greatly from patient to patient but was in all cases above the normal range.

Neonatal mortality was calculated in relation to peak serum CK-BB activity in the patients from the six groups. Data from

Table 4. $C K-B B$ activity (IU/liter) in serum of high-risk infants from $6 \mathrm{hr}$ to 15 days of age

\begin{tabular}{ccc}
\hline Group & & $n^{2}$ \\
\hline 1 & $4.7 \pm 5.2^{3}$ & 146 \\
2 & $3.7 \pm 4.6$ & 43 \\
3 & $7.6 \pm 11.0$ & 40 \\
4 & $20.4 \pm 23.5^{4}$ & 46 \\
5 & $2.5 \pm 2.6$ & 57 \\
\hline
\end{tabular}

${ }^{1}$ The groups are described in the text.

${ }^{2} n$, sample size.

${ }^{3}$ Mean \pm S.D.

${ }^{4} P<0.001$ (versus 1 ), Mann-Whitney test.

Table 5. $C K-M B$ and $C K-M M$ activity (IU/liter) in serum of healthy infants from $6 \mathrm{hr}$ to 15 days of age (group 1)

\begin{tabular}{rccc}
\hline Age & CK-MB & CK-MM & $n^{1}$ \\
\hline $6 \mathrm{hr}$ & $16.3 \pm 11.1^{2}$ & $172 \pm 57^{1}$ & 6 \\
$12 \mathrm{hr}$ & $17.1 \pm 14.9$ & $280 \pm 202$ & 25 \\
$24 \mathrm{hr}$ & $15.5 \pm 13.0$ & $263 \pm 281$ & 25 \\
$48 \mathrm{hr}$ & $12.7 \pm 9.9$ & $246 \pm 185$ & 24 \\
$72 \mathrm{hr}$ & $9.1 \pm 7.5$ & $157 \pm 103$ & 27 \\
$5 \mathrm{days}$ & $7.8 \pm 8.2$ & $104 \pm 63$ & 25 \\
15 days & $7.1 \pm 6.1$ & $88 \pm 45$ & 14 \\
\hline
\end{tabular}

${ }^{1} n$, sample size.

${ }^{2}$ Mean \pm S.D. 


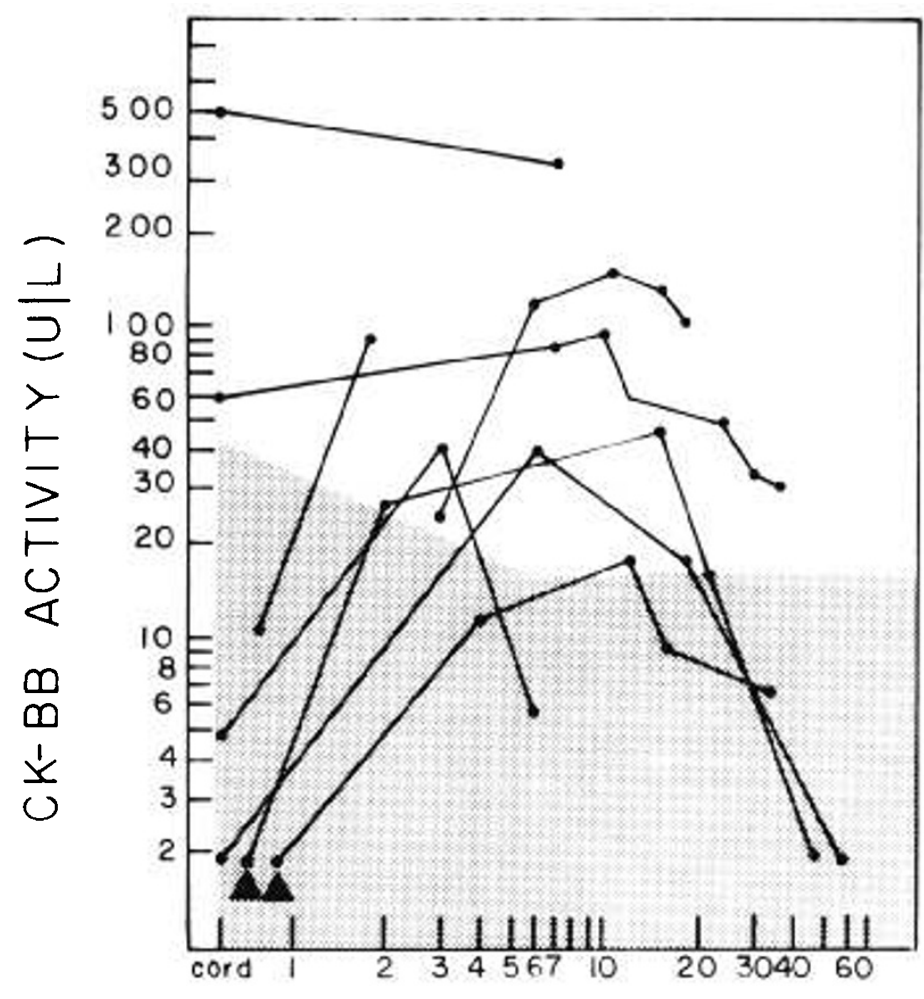

AGE (HOURS)

Fig. I. Serial measurements of CK-BB activity (IU/liter) in serum of five severely asphyxiated newborns and two infants with insertion of ventriculoperitoneal shunts $(\boldsymbol{\Delta})$. The initial samples in the latter were drawn one hr before surgery and not at birth. Dotted area, normal range for CK-BB activity as defined in the text.

patients with ventriculoperitoneal shunts were not included in these calculations. Peak CK-BB activity was defined as the mean of the three highest consecutive CK-BB results on each patient. There was an association between elevated peak CK-BB activity and neonatal mortality: when the peak CK-BB activity was higher than 35 IU/liter, the mortality was high (5 of 6); with peak CKBB levels between 16 and 35 IU/liter, the mortality was 3 of 10 ; and with peak CK-BB levels in the normal range (less than 16 IU/liter), the mortality was 4 of 45 . The difference between the first and the last groups was significant $\left(P<0.025 ; \chi^{2}\right.$ test corrected for continuity). The mortality was similar in groups 3 and $4: 4$ of 9 and 4 of 11 , respectively.

\section{DISCUSSION}

Serum CK-BB activity at birth and during the neonatal period in healthy premature newborns or newborns with minor problems was well above the lower limit of detectability of the method used, indicating that measurable levels of the isoenzyme may be found in sera from normal or nearly normal patients. This is in accord with other investigators $(1,4)$ and against the concept $(9-11,16)$ that CK-BB activity in serum is a pathologic finding. The findings of a high serum CK-BB at birth and a positive fetal-maternal gradient for CK-BB may both be due to leakage of CK-BB from the placenta, an organ whose CK-BB content is similar to that of brain (10), into the maternal and fetal circulations. This would raise serum CK-BB activity significantly in the fetus but only slightly in the mother because of differences in dilution. Laboda and Britton (10) have indeed found only a minor increase in maternal serum CK-BB activity during labor. Another explanation would be increased release of CK-BB from the fetal brain as result of intermittent compression and decompression of the fetal head during labor contractions. This effect would be magnified in infants of very low birth weight and maturity because of the higher vulnerability of their cranial structures to mechanical trauma and would be absent in babies who never went through labor. The higher cord serum CK-BB in infants who weighed less than $1750 \mathrm{~g}$ and the absence of a significant cord serum CK-BB elevation in newborns delivered by cesarean section without going through labor give support to this view.

The decline of serum CK-BB within $6 \mathrm{hr}$ of birth followed by a period of stable CK-BB levels would be the pattern expected after the infusion of the isoenzyme into the fetal circulation is interrupted at delivery; it is comparable in duration to the decline in serum CK-MB activity observed after the injection of that isoenzyme in dogs (19) and to the in vitro inactivation curve for human CK-BB (4).

Postasphyxial serum CK-BB activity was considerably higher in the newborns studied by Menzel and Thomas (13) (63.7 \pm 75.4 IU/liter) than in our patients $(20.2 \pm 23.8 \mathrm{IU} /$ liter $)$. The lower value in our series undoubtedly reflects the use of serial samples which included results in the normal range, plus the fact that Menzel and Thomas reported only data from patients known to have high serum CK activity. In another report Becker and Menzel (2) also showed marked elevations of serum CK-BB in asphyxiated newborns, but those elevations were presented only as semiquantitative data, and the patients were selected retrospectively on the basis of high serum CK activity. By doing quantitative determinations of $C K$ isoenzymes in serial samples obtained from prospectively defined groups, we demonstrated that a transient but significant elevation of serum CK-BB follows severe neonatal asphyxia. The correlation between the magnitude of this increase and neonatal mortality suggests a quantitative relationship between the extent of CK-BB elevation and the severity of cerebral damage in a manner analogous to the relation between serum CK-MB activity and myocardial infarction size. In spite of the presence of CK-BB in tissues other than brain, the elevation of serum CK-BB activity seemed to be associated exclusively with neuronal injury and was not observed in patients with other problems involving damage to various tissues. Our data also showed large increases of serum CK-MB and CK-MM in asphyxiated infants. A rise in serum CK-MB is to be expected because transient myocardial dysfunction and ischemia occur in stressed or asphyxiated newborns $(5,18)$; it would be reasonable to expect that skeletal muscle would also be affected, resulting in release of CK-MM.

Protection by barbiturates against hypoxic damage has been demonstrated conclusively in primates and other animal species $(3,7,8,12,14,15)$. In humans, Trolle $(22)$ found lower mortality in premature infants whose mothers were treated with phenobarbital for three days before delivery, but it was not specified whether the babies were asphyxiated. The protection by barbiturates occurs even when the drug is administered after the hypoxic event $(14,15)$ and may be due to decreased oxygen consumption (20) and shift to anaerobic metabolism or to other mechanisms (7). Inasmuch as the postasphyxial rise of serum CK-BB is an indicator of neuronal damage, the lack of such elevation in the pentobarbital-treated patients may be interpreted as protection by the drug against hypoxia. However, because the groups were small and the mortality was similar in groups 3 and 4 , we cannot conclude that such protection was clinically important. It is also possible that the hypoxic event was less severe in group 3 and thus failed to elicit a rise in CK-BB; however, if this were the case. one would have expected a much lower mortality in that group.

\section{REFERENCES AND NOTES}

I. Bayer, P. M., Gabl. F.. Gergely. T., and Zazgornik, J.: Isoenzyme der creatinkinase in der perinatalperiode. J. Clin. Chem. Clin. Biochem., 15: 349 (1975).

2. Becker, M., and Menzel, K.: Brain-typical creatinekinase in the serum of newborns with perinatal brain damage. Acta Paediatr. Scand., 67: 177 (1978).

3. Cockburn, F., Daniel. S.. Dawes, G. S.. James, L. S., Myers, R. E., Niemann. W.. Rodriguez, H., and Ross, B. B.: The effect of pentobarbital anesthesia or resuscitation and brain damage in fetal rhesus monkeys asphyxiated at delivery. J. Pediatr., 75: 281 (1969).

4. Fang, V. S.. Cho, H. W., and Meltzer, H. Y.: Radioimmunoassay for MM and 
BB isoenzymes of creatine kinase substantiated by clinical application. Clin. Chem., 23: 18 (1977).

5. Finley, J. P., Howan-Giles, R. B., Gildaj, D. L., Bloom, K. R., and Rowe, R. D.: Transient myocardial ischemia of the newborn infant demonstrated by thallium myocardial imaging. J. Pediatr., 94: 263 (1979).

6. Gilboa, N., and Swanson, R. J.: Serum creatine phosphokinase in normal newborns. Arch. Dis. Child., 51: 283 (1976)

7. Hakim, A. M., and Moss, G.: Cerebral effects of barbiturate: shift from "energy" to synthesis metabolism for cellular viability. Surg. Forum, 27: 497 (1976)

8. Hoff, J. T., Smith, A., Hankinson, H. L., and Nielsen, S. L.: Barbiturate protection from cerebral infarction in primates. Stroke, 6: 28 (1975).

9. Kaste, M., Somer, H., and Konttinen, A.: Brain-type creatine kinase isoenzyme. Occurrence in serum in acute cerebral disorders. Arch. Neurol., 34: 142 (1977).

10. Laboda, H. M., and Britton. V. J.: Creatine kinase isoenzyme activity in human placenta and in serum of women in labor. Clin. Chem., 23: 1329 (1977)

11. Lemberg, A., Werber, D. H., Gilardoni, A., and Magrino, H. J.: Presence of creatine kinase isoenzymes in liver failure with cerebral involvement. Clin. Chim. Acta, 80: 385 (1977).

12. McGraw, P.: Experimental cerebral infarction. Effects of pentobarbital in Mongolian gerbils. Arch. Neurol., 34: 334 (1977).

13. Menzel, K., and Thomas, A.: Gesamkaktivitat und Isoenzyme der Kreatinphosphokinase in Serum asphyktischer Neugeborener. Paediatr. Grenzgeb., 14: 5 (1975).

14. Michenfelder, J. D., Milde, J. H., and Sundt, T. M.: Cerebral protection by barbiturate anesthesia. Arch. Neurol., 33: 345 (1976).

15. Moseley, J., Laurent, J. P., and Molinari, G.: Barbiturate attenuation of the clinical course and pathologic lesions in a primate stroke model. Neurology, 25: 870 (1975).

Copyright (C) 1980 International Pediatric Research Foundation, Inc $0031-3998 / 80 / 1408-0935 \$ 02.00 / 0$
16. Nealon, D., and Henderson, A.: Measurement of brain-specific creatine kinase isoenzyme activity in serum. Clin. Chem., 21: 1663 (1975).

17. Nelson, R. M., Bucciarelli, R. L., Eitzman, D. V., Egan, E. A., and Gessner, I. H.: Serum creatine phosphokinase MB fraction in newborns with transient tricuspid insufficiency. N. Engl. J. Med., 298: 146 (1978).

18. Rowe, D. R., and Hoffman, T.: Transient myocardial ischemia of the newborm infant: a form of severe cardiorespiratory distress in full-term infants. J. Pediatr., 81: 243 (1972).

19. Sobel, B. E., Markham, J., Karlsberg, R. P., and Roberts, R. The nature of the disappearance of creatine kinase from the circulation and its influence of enzymatic estimation of infarct size. Circ. Res., 41: 836 (1977).

20. Sokoloff, L.: The action of drugs on the cerebral circulation. Pharmacol. Rev., II: 1 (1959).

21. Tietz, N. W.: Fundamentals of Clinical Chemistry. pp. $464-469$ (W. B. Saunders Co., Philadelphia, PA, 1970).

22. Trolle, D.: Decrease in mortality rates for low birth weight infants after phenobarbitone treatment. Acta Obstet. Gynecol. Scand., 55: 13 (1976).

23. Tsung, S. H.: Creatine kinase isoenzyme patterns in human tissue obtained at surgery. Clin. Chem., 22: 173 (1976).

24. Informed consent and approval by the Human Experimentation Committee was obtained for this study.

25. The author thanks Dr. David Werner and the personnel at the Pathologists' Laboratory and the Chemistry Laboratory at Schumpert Medical Center for their cooperation with this study.

26. Requests for reprints should be addressed to: Raul A. Cuestas, Jr., M.D., 915 Margaret Place, Shrevesport, LA 71101 (USA)

27. Received for publication May 14, 1979.

28. Accepted for publication December 21,1979 